\title{
On multivalued L-contractions and an application
}

\author{
M.A. Barakat ${ }^{1,2}$, Hassen Aydi ${ }^{3,4,5^{*}}$ (D), Aiman Mukheimer ${ }^{6}$, Ahmed A. Soliman ${ }^{1,7}$ and Abdallah Hyder ${ }^{7,8}$
}

\section{"Correspondence:}

hassen.aydi@tdtu.edu.vn

${ }^{3}$ Nonlinear Analysis Research Group,

Ton Duc Thang University, Ho Chi

Minh City, Vietnam

${ }^{4}$ Faculty of Mathematics and

Statistics, Ton Duc Thang University,

Ho Chi Minh City, Vietnam

Full list of author information is

available at the end of the article

\begin{abstract}
The aim of this paper is to present several fixed-point results for L-contractive multivalued mappings involving $\theta$-functions in the class of metric spaces. We also give some examples in support of the related concepts and presented results. A homotopy result is also provided.
\end{abstract}

MSC: Primary 46T99; 54E50; secondary 54H10

Keywords: Fixed point; Metric space; Multivalued mappings; L-contraction; $\theta$-function

\section{Introduction}

Fifty years ago, Nadler [1] introduced the idea of multivalued contraction mappings and presented his famous result, which generalized the Banach contraction principle [2] for multivalued mappings. In [3], the authors studied a problem of a global optimization using a common best proximity point of a pair of multivalued mappings. Also, Debnath and Srivastava [4] introduced a new and proper extension of Kannan's fixed point theorem to the case of multivalued maps using Wardowski's $F$-contraction. Going in same direction, several research works in fixed point theory related to multivalued contractions in different areas have appeared. For more details, see [4-23].

Let $(X, d)$ be a metric space and denote by $C B(X)$ the family of nonempty, bounded, and closed subsets of $X$. For $\Lambda_{1}, \Lambda_{2} \in C B(X)$, define $\mathcal{H}: C B(X) \times C B(X) \rightarrow[0, \infty)$ by

$$
\mathcal{H}\left(\Lambda_{1}, \Lambda_{2}\right)=\max \left\{\sup _{a \in \Lambda_{1}} d\left(a, \Lambda_{2}\right), \sup _{b \in \Lambda_{2}} d\left(b, \Lambda_{1}\right)\right\}
$$

where $d\left(a, \Lambda_{2}\right)=\inf \left\{d(a, \rho): \rho \in \Lambda_{2}\right\}$. Such a function $\mathcal{H}$ is called the Hausdorff-Pompieu metric induced by the metric $d$. Also, denote by $C L(X)$ the family of nonempty and closed subsets of $X$ and by $K(X)$ the family of nonempty and compact subsets of $X$.

On the other hand, a new type of a contraction mapping, known as an $\theta$-contraction, was introduced by Jleli and Samet [24].

(c) The Author(s) 2020. This article is licensed under a Creative Commons Attribution 4.0 International License, which permits use, sharing, adaptation, distribution and reproduction in any medium or format, as long as you give appropriate credit to the original author(s) and the source, provide a link to the Creative Commons licence, and indicate if changes were made. The images or other third party material in this article are included in the article's Creative Commons licence, unless indicated otherwise in a credit line to the material. If material is not included in the article's Creative Commons licence and your intended use is not permitted by statutory regulation or exceeds the permitted use, you will need to obtain permission directly from the copyright holder. To view a copy of this licence, visit http://creativecommons.org/licenses/by/4.0/. 
Definition 1.1 ([24]) Let $(X, d)$ be a metric space. A map $T: X \rightarrow X$ is said to be a $\theta$ contraction whenever there are $k \in(0,1)$ and $\theta \in \Theta$ such that

$$
\theta(d(T \varsigma, T \tau)) \leq[\theta(d(\varsigma, \tau))]^{k}
$$

for all $\varsigma, \tau \in X$ with $d(T \varsigma, T \tau)>0$, where $\Theta$ is the set of functions $\theta:(0, \infty) \rightarrow(1, \infty)$ verifying the following conditions:

$(\theta 1) \theta$ is nondecreasing;

$(\theta 2)$ for each positive sequence $\left\{t_{n}\right\}, \lim _{n \rightarrow \infty} \theta\left(t_{n}\right)=1 \mathrm{iff} \lim _{n \rightarrow \infty} t_{n}=0$;

$(\theta 3)$ there are $v \in(0, \infty]$ and $\mu \in(0,1)$ such that $\lim _{t \rightarrow 0^{+}} \frac{\theta(t)-1}{t^{\mu}}=v$.

Some years later, Vetro [23] introduced multivalued $\theta$-contraction mappings and gave the multivalued version of the main results of Jleli and Samet [24].

Definition 1.2 ([23]) A map $T: X \rightarrow C L(X)$ is said to be a weak $\theta$-contraction if there are $k \in(0,1)$ and $\theta \in \Theta$ such that

$$
\theta(\mathcal{H}(T \varsigma, T \tau)) \leq[\theta(d(\varsigma, \tau))]^{k}
$$

for all $\varsigma, \tau \in X$ with $\mathcal{H}(T \varsigma, T \tau)>0$.

Theorem 1.3 ([23]) Let $(X, d)$ be a complete metric space and $T: X \rightarrow K(X)$ be a weak $\theta$-contraction mapping. Then $T$ admits a fixed point.

Later in 2017, Ahmad et al. [25] replaced condition $(\theta 3)$ by the continuity condition of $\theta$ on $(0, \infty)$.

Example 1.4 ([25]) Note that $(\theta 3)$ and the continuity condition on $\theta$ are independent. Indeed, for $s>1$, the function given by $\theta(u)=e^{u^{s}}$ satisfies conditions $(\theta 1)$ and $(\theta 2)$, but it does not satisfy ( $\theta 3)$, while it is continuous. On the other hand, for all $s>1$ and $t \in\left(0, \frac{1}{s}\right)$, the function defined by $\theta(u)=1+u^{t}(1+[u])$, where $[u]$ denotes the integer part of $u$, satisfies conditions $(\theta 1),(\theta 2)$, and $(\theta 3)$ for each $k \in\left(\frac{1}{s}, 1\right)$, but it is not continuous. Also, the function $\theta(u)=e^{\sqrt{u}}$ is continuous and satisfies conditions $(\theta 1),(\theta 2)$, and $(\theta 3)$.

Now, denote by $\Theta^{*}$ the set of continuous functions satisfying $(\theta 1)$ and $(\theta 2)$. We observe that $\Theta \nsubseteq \Theta^{*}, \Theta^{*} \nsubseteq \Theta$, and $\Theta \cap \Theta^{*} \neq \emptyset$.

In [25], the authors gave the following fixed point theorem, extending the results of Jleli and Samet [24]. In [25] they considered $\theta \in \Theta^{*}$ instead of $\theta \in \Theta$.

Theorem 1.5 ([25]) Any $\theta$-contraction mapping $T: X \rightarrow X$ (with $\left.\theta \in \Theta^{*}\right)$ on a complete metric space $(X, d)$ possesses a unique fixed point.

Recently, Cho [26] initiated the concept L-simulation functions. Let $\xi:[1, \infty)^{2} \rightarrow \mathbb{R}$ verify the following assertions:

$\left(\xi_{1}\right) \xi(1,1)=1$

$\left(\xi_{2}\right) \xi(\tau, v)<\frac{v}{\tau}$ for all $v, \tau>1$; 
$\left(\xi_{3}\right)$ for all sequences $\left\{\tau_{n}\right\}$ and $\left\{v_{n}\right\}$ in $(1, \infty)$ with $\tau_{n}<v_{n}$ for $n=1,2,3, \ldots$,

$$
\lim _{n \rightarrow \infty} \tau_{n}=\lim _{n \rightarrow \infty} v_{n}>1 \Longrightarrow \limsup _{n \rightarrow \infty} \xi\left(\tau_{n}, v_{n}\right)<1 .
$$

Any $\xi \in \mathcal{L}$ is said an L-simulation function. Here, $\xi(\iota, \iota)<1$ for each $\iota>1$. As examples of $\mathrm{L}$-simulation functions, we state the following:

Example $1.6([26]) \xi(t, s)=\frac{s^{k}}{t}$ for all $s, t \geq 1$, where $k \in(0,1)$.

Example $1.7([26]) \xi(t, s)=\frac{s}{t \varphi(s)}$ for all $s, t \geq 1$ where $\varphi:[1, \infty) \rightarrow[1, \infty)$ is nondecreasing and lower semicontinuous such that $\varphi^{-1}(\{1\})=\{1\}$.

Example $1.8([26])$

$$
\xi(v, \eta)= \begin{cases}1 & \text { if }(v, \eta)=(1,1) \\ \frac{v}{2 \eta} & \text { if } v<\eta \\ \frac{v^{v}}{\eta} & \text { otherwise }\end{cases}
$$

for all $v, \eta \geq 1$ where $v \in(0,1)$.

This class of L-simulation functions is used as control functions in order to enrich the fixed point theory when dealing with several types of contraction mappings in variant (generalized) metric spaces. In this paper, based on L-simulation functions, we define a new type of multivalued contraction mappings, called multivalued L-contraction mappings via $\theta$-functions. We give some related fixed point results in the context of complete metric spaces. Some consequences are also derived. Moreover, an example to support our results is given. At the end, as an application, a homotopy result is provided.

\section{Main results}

Now, we introduce the definition of multivalued L-contraction mappings via $\theta$-functions.

Definition 2.1 Let $(X, d)$ be a metric space. A multivalued mapping $T: X \rightarrow C B(X)$ is called an L-contraction with respect to $\xi$ whenever there are $\theta \in \Theta^{*}$ and $\xi \in \mathcal{L}$ such that

$$
\xi(\theta(\mathcal{H}(T \varsigma, T \tau)), \theta(d(\varsigma, \tau))) \geq 1
$$

for all $\varsigma, \tau \in X$ with $\mathcal{H}(T \varsigma, T \tau)>0$.

Lemma 2.2 If $T: X \rightarrow C B(X)$ is an L-contraction with respect to $\xi$, then $T$ is continuous.

Proof Let $v \in X$ and $\left\{\sigma_{n}\right\} \subset X$ be such that

$$
\lim _{n \rightarrow \infty} d\left(\sigma_{n}, v\right)=0, \quad \text { and } \quad \mathcal{H}\left(T \sigma_{n}, T v\right)>0, \quad n \geq 0 .
$$

Since $T$ satisfies condition (2.1), we have

$$
1 \leq \xi\left(\theta\left(\mathcal{H}\left(T \sigma_{n}, T x\right)\right), \theta\left(d\left(\sigma_{n}, v\right)\right)\right)<\frac{\theta\left(d\left(\sigma_{n}, v\right)\right)}{\theta\left(\mathcal{H}\left(T \sigma_{n}, T v\right)\right)}, \quad \forall n=0,1,2, \ldots
$$


This implies that

$$
\theta\left(\mathcal{H}\left(T \sigma_{n}, T v\right)\right)<\theta\left(d\left(\sigma_{n}, v\right)\right), \quad \forall n=0,1,2, \ldots
$$

From $(\theta 1)$, one gets

$$
\mathcal{H}\left(T \sigma_{n}, T v\right)<d\left(\sigma_{n}, v\right), \quad \forall n=0,1,2, \ldots
$$

As $n \rightarrow \infty$, we find

$$
\lim _{n \rightarrow \infty} \mathcal{H}\left(T \sigma_{n}, T v\right)=0
$$

This proves that $T$ is continuous.

Theorem 2.3 Any L-contraction mapping $T: X \rightarrow C B(X)$ with respect to $\xi$ on a complete metric space $(X, d)$ possesses a fixed point.

Proof Let $\sigma_{0} \in X$ and $\sigma_{1} \in T \sigma_{0}$. If $\sigma_{0}=\sigma_{1}$, then $\sigma_{0}$ is a fixed point. If $\sigma_{1} \in T \sigma_{1}, \sigma_{1}$ is a fixed point. So, assume that $\sigma_{0} \neq \sigma_{1}$ and $\sigma_{1} \notin T \sigma_{1}$. We have

$$
0<d\left(\sigma_{1}, T \sigma_{1}\right) \leq \mathcal{H}\left(T \sigma_{0}, T \sigma_{1}\right)
$$

Since $T$ satisfies condition (2.1) and $\mathcal{H}\left(T \sigma_{0}, T \sigma_{1}\right)>0$, we have

$$
1 \leq \xi\left(\theta\left(\mathcal{H}\left(T \sigma_{0}, T \sigma_{1}\right)\right), \theta\left(d\left(\sigma_{0}, \sigma_{1}\right)\right)\right)<\frac{\theta\left(d\left(\sigma_{0}, \sigma_{1}\right)\right)}{\theta\left(\mathcal{H}\left(T \sigma_{0}, T \sigma_{1}\right)\right)}
$$

This implies that

$$
\theta\left(\mathcal{H}\left(T \sigma_{0}, T \sigma_{1}\right)\right)<\theta\left(d\left(\sigma_{0}, \sigma_{1}\right)\right)
$$

Using $(\theta 1)$, we get

$$
\mathcal{H}\left(T \sigma_{0}, T \sigma_{1}\right)<d\left(\sigma_{0}, \sigma_{1}\right)
$$

Since $\theta$ is continuous and nondecreasing, one has

$$
\inf _{y \in T \sigma_{1}} \theta\left(d\left(\sigma_{1}, y\right)\right)=\theta\left(d\left(\sigma_{1}, T \sigma_{1}\right)\right) \leq \theta\left(\mathcal{H}\left(T \sigma_{0}, T \sigma_{1}\right)\right)
$$

Hence, there exists $\sigma_{2} \in T \sigma_{1}$ such that

$$
\theta\left(d\left(\sigma_{1}, \sigma_{2}\right)\right) \leq \theta\left(\mathcal{H}\left(T \sigma_{0}, T \sigma_{1}\right)\right)
$$

By using $(\theta 1),(2.3)$, and (2.4), we obtain

$$
d\left(\sigma_{1}, \sigma_{2}\right) \leq \mathcal{H}\left(T \sigma_{0}, T \sigma_{1}\right)<d\left(\sigma_{0}, \sigma_{1}\right)
$$


If $\sigma_{2} \in T \sigma_{2}$, then $\sigma_{2}$ is a fixed point. Otherwise, proceeding similarly, there is $\sigma_{3} \in T \sigma_{2}$ such that

$$
d\left(\sigma_{2}, \sigma_{3}\right) \leq \mathcal{H}\left(T \sigma_{1}, T \sigma_{2}\right)<d\left(\sigma_{1}, \sigma_{2}\right)
$$

Similarly,

$$
0<d\left(\sigma_{n}, \sigma_{n+1}\right) \leq \mathcal{H}\left(T \sigma_{n-1}, T \sigma_{n}\right)<d\left(\sigma_{n-1}, \sigma_{n}\right), \quad \text { for all } n=1,2, \ldots
$$

Hence $\left\{d\left(\sigma_{n-1}, \sigma_{n}\right)\right\}$ is decreasing, and so there is $r \geq 0$ such that

$$
\lim _{n \rightarrow \infty} d\left(\sigma_{n-1}, \sigma_{n}\right)=r
$$

By (2.5), we have

$$
r=\lim _{n \rightarrow \infty} \mathcal{H}\left(T \sigma_{n-1}, T \sigma_{n}\right)=\lim _{n \rightarrow \infty} d\left(\sigma_{n-1}, \sigma_{n}\right)
$$

Assume that $r>0$. Using $(\theta 1)$ and the continuity of $\theta$, we get

$$
\lim _{n \rightarrow \infty} \theta\left(\mathcal{H}\left(T \sigma_{n-1}, T \sigma_{n}\right)\right)=\lim _{n \rightarrow \infty} \theta\left(d\left(\sigma_{n-1}, \sigma_{n}\right)\right)=\theta(r)>1 .
$$

Due to $(\xi 3)$, we have

$$
1 \leq \limsup _{n \rightarrow \infty} \xi\left(\theta\left(\mathcal{H}\left(T \sigma_{n-1}, T \sigma_{n}\right)\right), \theta\left(d\left(\sigma_{n-1}, \sigma_{n}\right)\right)\right)<1
$$

which is a contradiction. Therefore,

$$
\lim _{n \rightarrow \infty} d\left(\sigma_{n}, \sigma_{n+1}\right)=0
$$

Now, we will show that $\left\{\sigma_{i}\right\}$ is bounded in $(X, d)$.

If it is not the case, then there is a subsequence $\left\{\sigma_{i(q)}\right\}$ of $\left\{\sigma_{i}\right\}$ such that for $i(1)=1$ and for all $q=1,2, \ldots$, we have that $i(q+1)$ is the minimum integer greater than $i(q)$ with

$$
d\left(\sigma_{i(q+1)}, \sigma_{i(q)}\right)>1 \quad \text { and } \quad d\left(\sigma_{i(q)}, \sigma_{l}\right) \leq 1 \text {, }
$$

for all $i(q) \leq l \leq i(q+1)-1$. We have

$$
\begin{aligned}
1 & <d\left(\sigma_{i(q+1)}, \sigma_{i(q)}\right) \leq d\left(\sigma_{i(q+1)}, \sigma_{i(q+1)-1}\right)+d\left(\sigma_{i(q+1)-1}, \sigma_{i(q)}\right) \\
& \leq d\left(\sigma_{i(q+1)}, \sigma_{i(q+1)-1}\right)+1 .
\end{aligned}
$$

Letting $q \rightarrow \infty$ and using (2.7), we get

$$
\lim _{q \rightarrow \infty} d\left(\sigma_{i(q+1)}, \sigma_{i(q)}\right)=1
$$

Also,

$$
d\left(\sigma_{i(q+1)-1}, \sigma_{i(q)-1}\right) \leq d\left(\sigma_{i(q+1)-1}, \sigma_{i(q+1)}\right)+d\left(\sigma_{i(q+1)}, \sigma_{i(q)}\right)+d\left(\sigma_{i(q)}, \sigma_{i(q)-1}\right) .
$$


By taking the limit as $q \rightarrow \infty$, from (2.7) and (2.9), we have

$$
\lim _{q \rightarrow \infty} d\left(\sigma_{i(q+1)-1}, \sigma_{i(q)-1}\right) \leq 1
$$

Since

$$
\begin{aligned}
1 & =\lim _{q \rightarrow \infty} d\left(\sigma_{i(q+1)}, \sigma_{i(q)}\right) \leq \lim _{q \rightarrow \infty}\left[d\left(\sigma_{i(q+1)}, \sigma_{i(q+1)-1}\right)+d\left(\sigma_{i(q+1)-1}, \sigma_{i(q)-1}\right)+d\left(\sigma_{i(q)-1}, \sigma_{i(q)}\right)\right] \\
& \leq \lim _{q \rightarrow \infty} d\left(\sigma_{i(q+1)-1}, \sigma_{i(q)-1}\right) \leq 1,
\end{aligned}
$$

we obtain

$$
\lim _{q \rightarrow \infty} d\left(\sigma_{i(q+1)-1}, \sigma_{i(q)-1}\right)=1
$$

Again,

$$
1<d\left(\sigma_{i(q)}, \sigma_{i(q+1)}\right) \leq \mathcal{H}\left(T \sigma_{i(q)-1}, T \sigma_{i(q+1)-1}\right)
$$

So

$$
0<\mathcal{H}\left(T \sigma_{i(q)-1}, T \sigma_{i(q+1)-1}\right)
$$

Hence, using condition (2.1), we get

$$
1 \leq \xi\left(\theta\left(\mathcal{H}\left(T \sigma_{i(q+1)-1}, T \sigma_{i(q)-1}\right)\right), \theta\left(d\left(\sigma_{i(q+1)-1}, \sigma_{i(q)-1}\right)\right)\right)<\frac{d\left(\sigma_{i(q+1)-1}, \sigma_{i(q)-1}\right)}{\left.\mathcal{H}\left(T \sigma_{i(q+1)-1}, T \sigma_{i(q)-1}\right)\right)}
$$

One then gets

$$
\mathcal{H}\left(T \sigma_{i(q+1)-1}, T \sigma_{i(q)}-1\right)<d\left(\sigma_{i(q+1)-1}, \sigma_{i(q)-1}\right) .
$$

By the continuity of $\theta$, we have

$$
\begin{aligned}
\theta\left(d\left(\sigma_{i(q+1)}, T \sigma_{i(q)-1}\right)\right) & =\inf _{\sigma_{i(q)} \in T \sigma_{i(q)-1}} \theta\left(d\left(\sigma_{i(q+1)}, \sigma_{i(q)}\right)\right) \leq \theta\left(\mathcal{H}\left(T \sigma_{i(q+1)-1}, T \sigma_{i(q)-1}\right)\right) \\
& <\theta\left(d\left(\sigma_{i(q+1)-1}, \sigma_{i(q)-1}\right)\right) .
\end{aligned}
$$

By taking the limit as $q \rightarrow \infty$, we obtain

$$
\lim _{q \rightarrow \infty} \theta\left(\mathcal{H}\left(T \sigma_{i(q+1)-1}, T \sigma_{i(q)-1}\right)\right)=\lim _{q \rightarrow \infty} \theta\left(d\left(\sigma_{i(q+1)-1}, \sigma_{i(q)-1}\right)\right)=\theta(1)>1
$$

Due to ( $\xi 3)$, we get

$$
1 \leq \limsup _{q \rightarrow \infty} \xi\left(\theta\left(\mathcal{H}\left(T \sigma_{i(q+1)-1}, T \sigma_{i(q)-1}\right)\right), \theta\left(d\left(\sigma_{i(q+1)-1}, \sigma_{i(q)-1}\right)\right)\right)<1,
$$

which is a contradiction. Hence, $\left\{\sigma_{n}\right\}$ is bounded. 
We shall show the Cauchy property of $\left\{\sigma_{n}\right\}$. Let

$$
M_{i}=\sup \left\{d\left(\sigma_{k}, \sigma_{m}\right): k, m \geq i\right\} .
$$

It is clear that $0 \leq M_{i+1} \leq M_{i}<\infty$ for every $i=1,2,3, \ldots$. Thus, there is $M \geq 0$ such that $\lim _{i \rightarrow \infty} M_{i}=M$. Assume that $M>0$. Using (2.14), there exist $i(q), j(q) \geq q$ such that

$$
M_{q}-\frac{1}{q} \leq d\left(\sigma_{i(q)}, \sigma_{j(q)}\right) \leq M_{q} .
$$

Then

$$
\lim _{q \rightarrow \infty} d\left(\sigma_{i(q)}, \sigma_{j(q)}\right)=M
$$

By the triangle inequality, we have

$$
d\left(\sigma_{i(q)}, \sigma_{j(q)}\right) \leq d\left(\sigma_{i(q)}, \sigma_{i(q)-1}\right)+d\left(\sigma_{i(q)-1}, \sigma_{j(q)-1}\right)+d\left(\sigma_{j(q)-1}, \sigma_{j(q)}\right) .
$$

As $q \rightarrow \infty$, we find that

$$
\lim _{q \rightarrow \infty} d\left(\sigma_{i(q)-1}, \sigma_{j(q)-1}\right) \geq M .
$$

Also,

$$
\lim _{q \rightarrow \infty} d\left(\sigma_{i(q)-1}, \sigma_{j(q)-1}\right) \leq \lim _{q \rightarrow \infty}\left[d\left(\sigma_{i(q)-1}, \sigma_{i(q)}\right)+d\left(\sigma_{i(q)}, \sigma_{j(q)}\right)+d\left(\sigma_{j(q)}, \sigma_{j(q)-1}\right)\right] \leq M .
$$

From (2.15) and (2.16), we have

$$
\lim _{q \rightarrow \infty} d\left(\sigma_{i(q)-1}, \sigma_{j(q)-1}\right)=\lim _{q \rightarrow \infty} d\left(\sigma_{i(q)}, \sigma_{j(q)}\right)=M .
$$

Due to the continuity of $\theta$, we obtain

$$
\theta\left(d\left(\sigma_{i(q)}, T \sigma_{j(q)-1}\right)\right)=\inf _{\sigma_{j(q)} \in T \sigma_{j(q)-1}} \theta\left(d\left(\sigma_{i(q)}, \sigma_{j(q)}\right)\right) \leq \theta\left(\mathcal{H}\left(T \sigma_{i(q)-1}, T \sigma_{j(q)-1}\right)\right) .
$$

Letting $q \rightarrow \infty$, we have

$$
\theta(M) \leq \theta\left(\lim _{q \rightarrow \infty} \mathcal{H}\left(T \sigma_{i(q)-1}, T \sigma_{j(q)-1}\right)\right) .
$$

Applying $(\theta 1)$, one gets

$$
0<M \leq \lim _{q \rightarrow \infty} \mathcal{H}\left(T \sigma_{i(q)-1}, T \sigma_{j(q)-1}\right) .
$$

This implies that $\mathcal{H}\left(T \sigma_{i(q)-1}, T \sigma_{j(q)-1}\right)>0$. So by using condition (2.1), we have

$$
1 \leq \xi\left(\theta\left(\mathcal{H}\left(T \sigma_{i(q)-1}, T \sigma_{j(q)-1}\right)\right), \theta\left(d\left(\sigma_{i(q)-1}, \sigma_{j(q)-1}\right)\right)\right)<\frac{d\left(\sigma_{i(q)-1}, \sigma_{j(q)-1}\right)}{\left.\mathcal{H}\left(T \sigma_{i(q)-1}, T \sigma_{j(q)-1}\right)\right)}
$$


Hence,

$$
\theta\left(\mathcal{H}\left(T \sigma_{i(q+1)-1}, T \sigma_{j(q)-1}\right)\right)<\theta\left(d\left(\sigma_{i(q)-1}, \sigma_{j(q)-1}\right)\right) .
$$

Letting $q \rightarrow \infty$ and using $(\theta 1)$, we have

$$
\lim _{q \rightarrow \infty} \mathcal{H}\left(T \sigma_{i(q)-1}, T \sigma_{j(q)-1}\right)=\lim _{q \rightarrow \infty} d\left(\sigma_{i(q)-1}, \sigma_{j(q)-1}\right)=M
$$

In view of $(\xi 3)$, one gets

$$
1 \leq \limsup _{q \rightarrow \infty} \xi\left(\theta\left(\mathcal{H}\left(T \sigma_{i(q)-1}, T \sigma_{j(q)-1}\right)\right), \theta\left(d\left(\sigma_{i(q)-1}, \sigma_{j(q)-1}\right)\right)\right)<1,
$$

which is a contradiction.

Thus, $\lim _{q \rightarrow \infty} d\left(\sigma_{i(q)}, \sigma_{j(q)}\right)=0$, and hence $\left\{\sigma_{i}\right\}$ is a Cauchy sequence in the complete metric space $X$. Therefore, there is $y \in X$ such that

$$
\lim _{i \rightarrow \infty} d\left(\sigma_{i}, y\right)=0
$$

By Lemma 2.2, $T$ is continuous, and so

$$
0 \leq d(y, T y)=\lim _{i \rightarrow \infty} d\left(\sigma_{i}, T y\right) \leq \lim _{i \rightarrow \infty} \mathcal{H}\left(T \sigma_{i-1}, T y\right)=\mathcal{H}(T y, T y)=0
$$

This implies that $y$ is a fixed point of $T$.

We state some corollaries.

Corollary 2.4 Let $(X, d)$ be a complete metric space and $T: X \rightarrow C B(X)$ be a given mapping such that for all $\varsigma, \tau \in X$ with $\mathcal{H}(T \varsigma, T \tau) \neq 0$,

$$
\mathcal{H}(T \varsigma, T \tau) \leq d(\varsigma, \tau)-\varphi(d(\varsigma, \tau))
$$

where $\varphi:[0, \infty) \rightarrow[0, \infty)$ is lower semicontinuous and nondecreasing such that $\varphi^{-1}(\{0\})=$ $\{0\}$. Then $T$ admits a unique fixed point.

Proof From condition (2.18), we have

$$
e^{\mathcal{H}(T \varsigma, T \tau)} \leq e^{d(\varsigma, \tau)-\varphi(d(\varsigma, \tau)}
$$

Putting $\theta(t)=e^{t}$, we get

$$
\theta(\mathcal{H}(T \varsigma, T \tau)) \leq \frac{\theta(d(\varsigma, \tau))}{e^{\varphi(d(\varsigma, \tau))}}
$$

Also, define $\varphi(t)=\ln (\psi(\theta(t)))$, where $\psi:[1, \infty) \rightarrow[1, \infty)$ is lower semicontinuous and nondecreasing such that $\psi^{-1}(\{1\})=\{1\}$. We get

$$
\theta(\mathcal{H}(T \varsigma, T \tau)) \leq \frac{\theta(d(\varsigma, \tau))}{\psi(\theta(d(\varsigma, \tau)))}
$$


By putting $\xi(t, s)=\frac{s}{t \psi(s)}$, we get

$$
1 \leq \frac{\theta(d(\varsigma, \tau))}{\theta(\mathcal{H}(T \varsigma, T \tau)) \psi(\theta(d(\varsigma, \tau)))}=\xi(\theta(\mathcal{H}(T \varsigma, T \tau)), \theta(d(\varsigma, \tau))) .
$$

In view of Theorem 2.3, $T$ has a unique fixed point.

Now, take in Theorem 2.3 the function $\xi(v, \eta)=\frac{v^{\kappa}}{\eta}$ for all $v, \eta \geq 1$, where $\kappa \in(0,1)$.

Corollary 2.5 Let $(X, d)$ be a complete metric space and $T: X \rightarrow K(X)$ be such that for all $\varsigma, \tau \in X$ with $\mathcal{H}(T \varsigma, T \tau) \neq 0$,

$$
\theta(\mathcal{H}(T \varsigma, T \tau)) \leq[\theta(d(\varsigma, \tau))]^{k}
$$

where $\theta \in \Theta^{*}$. Then $T$ possesses a unique fixed point.

Corollary 2.6 Let $(X, d)$ be a complete metric space and $T: X \rightarrow C B(X)$ be such that for all $\varsigma, \tau \in X$ with $\mathcal{H}(T \varsigma, T \tau) \neq 0$,

$$
\theta(\mathcal{H}(T \varsigma, T \tau)) \leq[\theta(d(\varsigma, \tau))]^{k}
$$

where $\theta \in \Theta^{*}$ satisfies ( $\left.\theta 4\right)$. Then $T$ admits a unique fixed point.

Remark 2.7 Corollary 2.4 is the multivalued version of Theorem 3.2 in [24], and improves it by replacing the compact range condition by the closed and bounded range, and by considering that $\varphi$ as lower semicontinuous and not necessary continuous. Corollary 2.5 is the multivalued version of Theorem 1.5. Also, Corollary 2.6 is an extension of Theorem 2.5 in [23] without condition $\left(\theta_{3}\right)$.

The following example supports Theorem 2.3. Here, the main result of Vetro [23] is not applicable.

Example 2.8 Let $X=\{0,1,2,4\}$ be a metric space endowed with the metric $d(\varsigma, \tau)=|\varsigma-\tau|$ for all $\varsigma, \tau \in X$. Consider the mapping $T: X \rightarrow K(X)$ given by

$$
T \varsigma= \begin{cases}\{0\}, & \varsigma=4, \\ \{0,2\}, & \varsigma \neq 4 .\end{cases}
$$

Take $\theta(t)=e^{t}$ for each $t>0$. Choose $\xi(t, s)=\frac{s}{t \phi(s)}$ for all $t, s \geq 1$, where

$$
\phi(s)= \begin{cases}1 & \text { if } s \leq e^{2} \\ \sqrt[3]{s} & \text { if } s>e^{2}\end{cases}
$$

We shall prove that $T$ is a multivalued L-contraction with respect to such $\xi$. We notice that $\mathcal{H}(T \varsigma, T \tau)>0$ iff $\varsigma=4$ and $\tau \neq 4$. In this case, we have $\theta(\mathcal{H}(T \varsigma, T \tau))=\theta(\mathcal{H}(\{0\},\{0,2\}))=$ $e^{2}$. We need the following: 
Case 1 . If $\varsigma=4$ and $\tau=0$, then $\theta(d(4,0))=e^{4}$ and hence

$$
\xi(\theta(\mathcal{H}(T 4, T 0)), \theta(d(4,0)))=\frac{e^{4}}{e^{\frac{10}{3}}}=e^{\frac{2}{3}}>1
$$

Case 2. If $\varsigma=4$ and $\tau=1$, then $\theta(d(4,1))=e^{3}$. So,

$$
\xi(\theta(\mathcal{H}(T 4, T 1)), \theta(d(4,1)))=\frac{e^{3}}{e^{2} \sqrt[3]{e^{3}}}=1
$$

Case 3. If $\varsigma=4$ and $\tau=2$, then $\theta(d(4,2))=e^{2}$ and so

$$
\xi(\theta(\mathcal{H}(T 4, T 2)), \theta(d(4,2)))=\frac{e^{2}}{e^{2}(1)}=1 .
$$

Then $T$ is a multivalued L-contraction with respect to $\xi$. Hence all the conditions of Theorem 2.3 hold. Here, $T$ admits a fixed point.

Note that for all $\theta \in \Theta$, we have

$$
\theta(\mathcal{H}(T 4, T 2))=\theta(2)>[\theta(d(4,2))]^{k}=[\theta(2)]^{k} \quad \text { for all } \kappa \in[0,1) .
$$

That is, $T$ is not an $\theta$-contraction of Vetro [23].

\section{Application}

Now, we present a homotopy result as an application of Corollary 2.4.

Theorem 3.1 Let $(X, d)$ be a complete metric space, $E \subset X$ be a nonempty open set and let $D \subset X$ be a closed set with $E \subset D$. Also, let $T: D \times[0,1] \rightarrow C B(X)$ verify condition (2.18) such that

(i) $s \notin T(s, u)$, for all $s \in D \backslash E, u \in[0,1]$;

(ii) there is a continuous function $\gamma:[0,1] \rightarrow \mathbb{R}$ such that for all $u, v \in[0,1]$ and $s \in D$,

$$
\mathcal{H}(T(s, u), T(s, v)) \leq \lambda|\gamma(u)-\gamma(v)|, \quad \text { where } \lambda \in(0,1)
$$

(iii) if $s \in T(s, u)$, then $T(s, u)=\{s\}$.

Then $T(\cdot, 0)$ possesses a fixed point iff $T(\cdot, 1)$ possesses a fixed point.

Proof Let $A=\{u \in[0,1] ; s \in T(s, u)$, for some $s \in E\}$. Since $T(\cdot, 0)$ has a fixed point and from condition (i), we have $0 \in A$, therefore $A$ is nonempty. We claim that $A$ is both open and closed in $[0,1]$, then by the connectedness of $[0,1]$, the proof is completed.

First, we show that $A$ is open in $[0,1]$. Let $u_{0} \in A$, then there is $s_{0} \in E$ with $s_{0} \in T\left(s_{0}, u_{0}\right)$.

Since $E$ is open in $(X, d)$, there is $r>0$ such that $B\left(s_{0}, r\right) \subset E$. Take $\epsilon=\frac{\varphi(r)}{\lambda}>0$, where $\varphi$ is given in Corollary 2.4. Using the continuity of $\gamma$ at $u_{0}$, there is $\delta(\epsilon)>0$ such that $\left|\gamma(u)-\gamma\left(u_{0}\right)\right|<\epsilon$, for all $u \in\left(u_{0}-\delta, u_{0}+\delta\right)$.

Let $u \in\left(u_{0}-\delta, u_{0}+\delta\right)$. For $s \in B\left[s_{0}, r\right]=\left\{s \in X ; d\left(s, s_{0}\right) \leq r\right\}$, we get

$$
\begin{aligned}
d\left(T(s, u), s_{0}\right) & \leq \mathcal{H}\left(T(s, u), T\left(s_{0}, u_{0}\right)\right) \\
& \leq \mathcal{H}\left(T(s, u), T\left(s, u_{0}\right)\right)+\mathcal{H}\left(T\left(s, u_{0}\right), T\left(s_{0}, u_{0}\right)\right) .
\end{aligned}
$$


If $\mathcal{H}\left(T\left(s, u_{0}\right), T\left(s_{0}, u_{0}\right)\right)=0$, then using (iii),

$$
d\left(T(s, u), s_{0}\right) \leq \lambda\left|\gamma(u)-\gamma\left(u_{0}\right)\right|<\lambda \epsilon<r .
$$

In the case that $\mathcal{H}\left(T\left(s, u_{0}\right), T\left(s_{0}, u_{0}\right)\right)>0$ and since $T$ satisfies the contraction condition (2.18), we obtain

$$
\mathcal{H}\left(T\left(s, u_{0}\right), T\left(s_{0}, u_{0}\right)\right) \leq d\left(s, s_{0}\right)-\varphi\left(d\left(s, s_{0}\right)\right) .
$$

By substituting in (3.1) from (3.3) and from (iii), we have

$$
\begin{aligned}
d\left(T(s, u), s_{0}\right) & \leq \lambda\left|\gamma(u)-\gamma\left(u_{0}\right)\right|+d\left(s, s_{0}\right)-\varphi\left(d\left(s, s_{0}\right)\right) \\
& <\lambda \epsilon+r-\varphi(r)=r .
\end{aligned}
$$

Combining (3.2) and (3.4), and for all $u \in\left(u_{0}-\delta, u_{0}+\delta\right)$, the operator $T(\cdot, u): B\left[s_{0}, r\right] \rightarrow$ $C B(X)$ satisfies all hypotheses of Corollary 2.5. Hence, $T\left(\cdot, u_{0}\right)$ has a fixed point in $B\left[s_{0}, r\right] \subset$ $D$, and since (i) holds, this fixed point has to lie in $E$, and $\left(u_{0}-\delta, u_{0}+\delta\right) \subset A$. Therefore, $A$ is open.

Next, we prove that $A$ is closed. Let $\left\{u_{n}\right\}$ be a sequence in $A$ such that $\lim _{n \rightarrow \infty} u_{n}=\bar{u} \in$ $[0,1]$. Since $\left\{u_{n}\right\} \subset A$, there is $\left\{s_{n}\right\} \subset E$ such that $s_{n} \in T\left(s_{n}, u_{n}\right)$. Then, by using condition (iii) and for all $m, n \in \mathbb{Z}^{+}$, we have

$$
\begin{aligned}
d\left(s_{n}, s_{m}\right) & \leq \mathcal{H}\left(T\left(s_{n}, u_{n}\right), T\left(s_{m}, u_{m}\right)\right) \\
& \leq \mathcal{H}\left(T\left(s_{n}, u_{n}\right), T\left(s_{n}, u_{m}\right)\right)+\mathcal{H}\left(T\left(s_{n}, u_{m}\right), T\left(s_{m}, u_{m}\right)\right) .
\end{aligned}
$$

Case 1. If $\mathcal{H}\left(T\left(s_{n}, u_{m}\right), T\left(s_{m}, u_{m}\right)\right)=0$ for some $m, n \in \mathbb{Z}^{+}$, then from condition (ii) we have

$$
d\left(s_{n}, s_{m}\right) \leq \lambda\left|\gamma\left(u_{n}\right)-\gamma\left(u_{m}\right)\right|
$$

Case 2. If $\mathcal{H}\left(T\left(s_{n}, u_{m}\right), T\left(s_{m}, u_{m}\right)\right)>0$, then from the contraction condition (2.18), we have

$$
\mathcal{H}\left(T\left(s_{n}, u_{m}\right), T\left(s_{m}, u_{m}\right)\right) \leq d\left(s_{n}, s_{m}\right)-\varphi\left(d\left(s_{n}, s_{m}\right)\right) .
$$

By substituting (3.7) and condition (iii) in (3.5), we have

$$
d\left(s_{n}, s_{m}\right) \leq \lambda\left|\gamma\left(u_{n}\right)-\gamma\left(u_{m}\right)\right|+d\left(s_{n}, s_{m}\right)-\varphi\left(d\left(s_{n}, s_{m}\right)\right) .
$$

From (3.6) and (3.8), we have

$$
d\left(s_{n}, s_{m}\right) \leq \lambda\left|\gamma\left(u_{n}\right)-\gamma\left(u_{m}\right)\right|+d\left(s_{n}, s_{m}\right)-\varphi\left(d\left(s_{n}, s_{m}\right)\right),
$$

for each $m, n \in \mathbb{Z}^{+}$. Hence

$$
\varphi\left(d\left(s_{n}, s_{m}\right)\right) \leq \lambda\left|\gamma\left(u_{n}\right)-\gamma\left(u_{m}\right)\right| .
$$


By taking the limit as $n, m \rightarrow \infty$ and from the lower semicontinuity of $\varphi$ and continuity of $\gamma$, we get

$$
\lim _{n, m \rightarrow \infty} d\left(s_{n}, s_{m}\right)=0 .
$$

Hence, the sequence $\left\{s_{n}\right\}$ is Cauchy in $(X, d)$, which is complete, so there is $\bar{s} \in D$ with $\lim _{n \rightarrow \infty} d\left(s_{n}, \bar{s}\right)=0$. We have

$$
\begin{aligned}
d\left(s_{n}, T(\bar{s}, \bar{u})\right) & \leq \mathcal{H}\left(T\left(s_{n}, u_{n}\right), T(\bar{s}, \bar{u})\right) \\
& \leq \mathcal{H}\left(T\left(s_{n}, u_{n}\right), T\left(s_{n}, \bar{u}\right)\right)+\mathcal{H}\left(T\left(s_{n}, \bar{u}\right), T(\bar{s}, \bar{u})\right) .
\end{aligned}
$$

Case 1. If $\mathcal{H}\left(T\left(s_{n}, \bar{u}\right), T(\bar{s}, \bar{u})\right)=0$, for some $n$ then from condition (ii) we have

$$
d\left(s_{n}, T(\bar{s}, \bar{u})\right) \leq \lambda\left|\gamma\left(u_{n}\right)-\gamma(\bar{u})\right| .
$$

Case 2. If $\mathcal{H}\left(T\left(s_{n}, \bar{u}\right), T(\bar{s}, \bar{u})\right)>0$ for each $n$, then from the contraction condition (2.18), we have

$$
\mathcal{H}\left(T\left(s_{n}, \bar{u}\right), T(\bar{s}, \bar{u})\right) \leq d\left(s_{n}, \bar{s}\right)-\varphi\left(d\left(s_{n}, \bar{s}\right)\right) .
$$

By substituting from (3.11) and condition (iii) in (3.9), we have

$$
d\left(s_{n}, T(\bar{s}, \bar{u})\right) \leq \lambda\left|\gamma\left(u_{n}\right)-\gamma(\bar{u})\right|+d\left(s_{n}, \bar{s}\right)-\varphi\left(d\left(s_{n}, \bar{s}\right)\right) .
$$

By (3.10) and (3.12), we get that

$$
d\left(s_{n}, T(\bar{s}, \bar{u})\right) \leq \lambda\left|\gamma\left(u_{n}\right)-\gamma(\bar{u})\right|+d\left(s_{n}, \bar{s}\right),
$$

for each $n \in \mathbb{Z}^{+}$. By taking the limit as $n \rightarrow \infty$ and from continuity of $\gamma$, we obtain that

$$
\lim _{n \rightarrow \infty} d\left(s_{n}, T(\bar{s}, \bar{u})\right)=d(\bar{s}, T(\bar{s}, \bar{u}))=0 .
$$

We deduce that $\bar{s} \in T(\bar{s}, \bar{u})$, and by using condition (i), we have $\bar{s} \in E$. Thus, $\bar{u} \in A$, and so $A$ is a closed subset of $[0,1]$.

Similarly, we can deduce the reverse implication.

\section{Acknowledgements}

The authors thank anonymous referees for their remarkable comments, suggestions, and ideas that helped improve this paper. The third author would like to thank Prince Sultan University for funding this work through research group Nonlinear Analysis Methods in Applied Mathematics (NAMAM) group number RG-DES-2017-01-17.

\section{Funding}

This work is funded by Prince Sultan University through the research group Nonlinear Analysis Methods in Applied Mathematics (NAMAM) group number RG-DES-2017-01-17.

Availability of data and materials

No data were used to support this study. It is not applicable for our paper. 
Authors' contributions

All authors contributed equally and significantly in writing this article. All authors have read and agreed to the published version of the manuscript.

\section{Author details}

${ }^{1}$ Department of Mathematics, Faculty of Sciences, Al-Azhar University, 71524 Assiut, Egypt. ${ }^{2}$ Department of Mathematics, College of AI Wajh, University of Tabuk, Tabuk, Saudi Arabia. ${ }^{3}$ Nonlinear Analysis Research Group, Ton Duc Thang University, Ho Chi Minh City, Vietnam. ${ }^{4}$ Faculty of Mathematics and Statistics, Ton Duc Thang University, Ho Chi Minh City, Vietnam. ${ }^{5}$ Department of Medical Research, China Medical University Hospital, China Medical University, Hsueh-Shih Road, 40402 Taichung, Taiwan. ${ }^{6}$ Department of Mathematics and General Sciences, Prince Sultan University, Riyadh, 11586, Saudi Arabia. ${ }^{7}$ College of Science, Department of Mathematics, King Khalid University, P.O. Box 9004, 61413, Abha, Saudi Arabia. ${ }^{8}$ Department of Engineering Mathematics and Physics, Faculty of Engineering, Al-Azhar University, Cairo, Egypt.

\section{Publisher's Note}

Springer Nature remains neutral with regard to jurisdictional claims in published maps and institutional affiliations.

Received: 6 July 2020 Accepted: 28 September 2020 Published online: 06 October 2020

\section{References}

1. Nadler, S.B.: Multi-valued contraction mappings. Pac. J. Math. 30, 475-488 (1969)

2. Banach, S.: Sur les opérations dans les ensembles abstraits et leur application aux équations intégrales. Fundam. Math. 3, 133-181 (1922)

3. Debnath, P., Srivastava, H.M.: Global optimization and common best proximity points for some multivalued contractive pairs of mappings. Axioms 9, Article ID 102 (2020)

4. Debnath, P., Srivastava, H.M.: New extensions of Kannan's and Reich's fixed point theorems for multivalued maps using Wardowski's technique with application to integral equations. Symmetry 12, 1090 (2020)

5. Srivastava, H.M., Shehata, A., Moustafa, S.I.: Some fixed point theorems for $F(\psi, \phi)$-contractions and their application to fractional differential equations. Russ. J. Math. Phys. 27, 385-398 (2020)

6. Alamgir, N., Kiran, Q., Isik, H., Aydi, H.: Fixed point results via a Hausdorff controlled type metric. Adv. Differ. Equ. 2020, 24 (2020)

7. Ameer, E., Aydi, H., Arshad, M., De la Sen, M.: Hybrid Ćirić type graphic $(\Upsilon, \Lambda)$-contraction mappings with applications to electric circuit and fractional differential equations. Symmetry 12(3), 467 (2020)

8. Patle, P., Patel, D., Aydi, H., Radenovic, S.: On $\mathrm{H}^{+}$-type multivalued contractions and applications in symmetric and probabilistic spaces. Mathematics 7(2), 144 (2019)

9. Eldred, A.A., Anuradha, J., Veeramani, P.: On equivalence of generalized multi-valued contractions and Nadler's fixed point theorem. J. Math. Anal. Appl. 336(2), 751-757 (2007)

10. Debnath, P., Choudhury, B.S., Neog, M.: Fixed points of contractive set valued mappings with set valued domains on a metric space with graph. Fixed Point Theory Appl. 2017, Article number 5 (2016)

11. Ameer, E., Aydi, H., Arshad, M., Alsamir, H., Noorani, M.S.: Hybrid multivalued type contraction mappings in $\alpha_{K}$-complete partial b-metric spaces and applications. Symmetry 11(1), 86 (2019)

12. Aydi, H., Barakat, M.A., Mitrovic, Z.D., Cavic, V.S.: A Suzuki-type multivalued contraction on weak partial metric spaces and applications. J. Inequal. Appl. 2018, 270 (2018)

13. Altun, I., Minak, G.: On fixed point theorems for multivalued mappings of Feng-Liu type. Bull. Korean Math. Soc. 52(6) 1901-1910 (2015)

14. Aydi, H., Abbas, M., Vetro, C.: Partial Hausdorff metric and Nadler's fixed point theorem on partial metric spaces. Topol. Appl. 159, 3234-3242 (2012)

15. Debnath, P., De La Sen, M.: Fixed-points of eventually $\triangle$-restrictive and $\Delta(\epsilon)$-restrictive set-valued maps in metric spaces. Symmetry 12(1), 127 (2020)

16. Aydi, H., Abbas, M., Vetro, C.: Common fixed points for multivalued generalized contractions on partial metric spaces. Rev. R. Acad. Cienc. Exactas Fís. Nat., Ser. A Mat. 108, 483-501 (2014)

17. Aydi, H., Felhi, A., Karapinar, E., Sahmim, S.: A Nadler-type fixed point theorem in dislocated spaces and applications. Miskolc Math. Notes 19, 111-124 (2018)

18. Ciric, Lj.B.: Multi-valued nonlinear contraction mappings. Nonlinear Anal. 71, 2716-2723 (2009)

19. Afshari, H., Aydi, H., Karapınar, E.: On generalized $\alpha-\psi$-Geraghty contractions on b-metric spaces. Georgian Math. J. 27(1), 9-21 (2020)

20. Feng, Y., Liu, S.: Fixed point theorems for multi-valued contractive mappings and multi-valued Caristi type mappings. J. Math. Anal. Appl. 317, 103-112 (2006)

21. Kamran, T.: Mizoguchi-Takahashi's type fixed point theorem. Comput. Math. Appl. 57, 507-511 (2009)

22. Qawaqneh, H., Noorani, M.S., Shatanawi, W., Aydi, H., Alsamir, H.: Fixed point results for multi-valued contractions in b-metric spaces. Mathematics 7(2), 132 (2019)

23. Vetro, F.: A generalization of Nadler fixed point theorem. Carpath. J. Math. 31, 403-410 (2015)

24. Jeli, M., Samet, B.: New generalization of the Banach contraction principle. J. Inequal. Appl. 2014, 38 (2014)

25. Ahmad, J., Al-Mazrooei, A.E., Cho, Y.J., Yang, Y.O.: Fixed point results for generalized $\Theta$-contractions. J. Nonlinear Sci. Appl. 10, 2350-2358 (2017)

26. Cho, S.H.: Fixed point theorems for L-contractions in generalized metric spaces. Abstr. Appl. Anal. 2018, Article ID 1327691 (2018) 